\title{
BioéthiqueOnline
}

\section{Plaidoyer pour une reconnaissance de l'enfant comme agent moral : une réponse à Verpaelst et Couture}

\author{
Aliya O. Affdal
}

Volume 6, 2017

URI : https://id.erudit.org/iderudit/1044612ar

DOI : https://doi.org/10.7202/1044612ar

Aller au sommaire du numéro

Éditeur(s)

BioéthiqueOnline

ISSN

1923-2799 (numérique)

Découvrir la revue

Citer cet article

Affdal, A. O. (2017). Plaidoyer pour une reconnaissance de l'enfant comme agent moral : une réponse à Verpaelst et Couture. BioéthiqueOnline, 6 .

https://doi.org/10.7202/1044612ar
Résumé de l'article

Bien qu'il soit un être vulnérable, l'enfant pourrait être considéré comme un agent moral dans certaines circonstances. Ainsi, il peut et devrait être davantage associé aux décisions médicales le concernant. 


\title{
Plaidoyer pour une reconnaissance de l'enfant comme agent moral : Une réponse à Verpaelst et Couture
}

\author{
RÉPONSE À - TRAVAIL CRÉATIF / RESPONSE TO - CREATIVE WORK \\ Aliya O. Affdal $\left.\right|^{1,2,3}$
}

\begin{abstract}
Reçu/Received: 10 Dec $2016 \quad$ Publié/Published: 9 May 2017
Éditeurs/Editors: Hazar Haidar \& Renaud Boulanger

Travail créatif discuté/Creative Work discussed: Verpaelst F. A Very embarrassing moment. BioéthiqueOnline, 2015. 4(7);

Couture $\mathrm{V}$. La reconnaissance d'autrui comme prérequis à toute forme de consentement et d'assentiment: Une réponse à

Verpaelst et Touyz. BioéthiqueOnline, 2015. 4(17).
\end{abstract}

2017 AO Affdal, Creative Commons Attribution 4.0 International License

\section{Résumé}

Bien qu'il soit un être vulnérable, l'enfant pourrait être considéré comme un agent moral dans certaines circonstances. Ainsi, il peut et devrait être davantage associé aux décisions médicales le concernant.

\section{Mots clés}

enfant, vulnérabilité, agent moral, décisions médicales

\section{Summary}

Although a vulnerable human being, a child can be considered as a moral agent in certain circumstances. Thus, a child can and should be more involved in medical decisions about him/herself.

\section{Keywords}

child, vulnerability, moral agent, medical decisions

Affiliations des auteurs / Author Affiliations

${ }^{1}$ Programmes de bioéthique, Département de médecine sociale et préventive, École de Santé Publique de l'Université de Montréal (ESPUM), Montréal, Québec, Canada

${ }^{2}$ Institut de Recherche en Santé Publique de l'Université de Montréal (IRSPUM), Montréal, Canada

${ }^{3}$ Centre de Recherche du Centre hospitalier de l'Université de Montréal (CRCHUM), Montréal, Québec, Canada

\section{Correspondance / Correspondence}

Aliya O. Affdal, oulaya.affdal@umontreal.ca

\section{Remerciements}

L'auteure tient à remercier les éditeurs Hazar Haidar et Renaud Boulanger pour leurs commentaires pertinents.

\section{Conflit d'intérêts}

L'auteure est éditrice de la section des Commentaires pour la revue BioéthiqueOnline; elle n'a participé à aucune étape du processus d'évaluation.

\section{Acknowledgements}

The author would like to thank the editors Hazar Haidar and Renaud Boulanger for their helpful comments.

\section{Conflicts of Interest}

The author is Section editor for Commentaries at BioéthiqueOnline; she was not involved in any part of the evaluation process.

\section{Introduction}

Dans son manuscrit, Frank Verpaelst relate son expérience alors qu'il était, malgré lui, mannequin de formation à l'hôpital à l'âge de 7 ans [1]. II met en relief deux aspects très importants en éthique : la vulnérabilité de l'enfant et le consentement du patient. Dans sa réponse à l'auteur, Vincent Couture avance qu' " un processus de consentement ou d'assentiment est éthiquement vain s'il n'est pas accompagné d'une reconnaissance authentique de cette personne " [2]. II se réfère à la théorie de l'auteur Honneth pour qui la reconnaissance peut être comprise comme un type de devoir envers autrui fait d'adresses et d'égards qui permettent de reconnaître l'individualité de l'Autre [3]. Dans ce commentaire, nous proposons de développer une seconde piste de réflexion en nous basant sur la reconnaissance d'une capacité à agir moralement et en nous posant la question suivante: la reconnaissance de l'enfant comme agent moral dépend-elle de son âge? Bien qu'il n'existe pas de définition communément admise, de façon générale « a moral agent [is] one who qualifies generally as an agent open to responsibility ascriptions. " [4] 
Dépendamment du contexte, tout acte médical requiert un consentement respectant les volontés du patient. Le consentement est considéré comme libre et éclairé lorsqu'il est volontaire (i.e., sans coercition). Pour remplir cette condition, la personne consentante doit recevoir toutes les informations nécessaires à sa prise de décision et doit être en pleine possession de ses facultés afin de comprendre ces informations [5]. Dans le cas particulier des enfants, la faculté à consentir est partiellement déterminée par le développement lié à l'âge. Par exemple, au Québec, des mineurs de 14 ans sont considérés aptes à consentir à certains soins. Lorsqu'ils ne le sont pas, les parents du/de la patient(e) ou les tuteurs légaux consentent au nom de l'enfant et son assentiment, plutôt qu'un consentement complet, peut être sollicité. Généralement, et contrairement au domaine de la recherche médicale, la pratique clinique actuelle encourage les efforts afin d'obtenir l'assentiment de l'enfant pour un traitement, mais cet assentiment n'est pas obligatoire [6].

Dans certains pays, comme aux États-Unis [7] ou en France [8], les enfants de moins de 18 ans ne sont pas légalement tenus de fournir un consentement éclairé. Pourtant, la rationalité et la compétence n'arrivent pas comme par magie le jour des 18 ans d'une personne [9]. En pédiatrie, il est reconnu que le niveau de développement cognitif et moral est variable même au sein d'un même groupe d'âge [10]. La maturité cognitive et morale n'apparaît pas dans toute sa perfection à un stade du développement de l'enfant [11]. Le cas de la transition des patients d'un service pédiatrique vers un service pour adultes en est un exemple. Dans le cas de la France, cette transition des soins se pose dès l'âge de 15 ans et 3 mois, âge administratif dans les hôpitaux, qui clôt légalement l'âge pédiatrique [12]. Pourtant, les médecins sont d'accord pour dire que le passage ne devrait pas dépendre seulement de l'âge du patient et que chacun d'eux a sa particularité. D'ailleurs dans la pratique, certains patients passent dans le service pour adultes à l'âge de 23 ans, alors que d'autres ont les capacités d'être transférés même avant 15 ans [13].

De nombreuses études soulignent que certains enfants, même très jeunes, ont des capacités proches de celles des adultes, comme le souhait de recevoir l'information les concernant. Pour exemple, voici quelques phrases de jeunes patients relevées dans un article autour de l'importance de reconnaître les ressentis des enfants en milieu pédiatrique [14] :

My wish is for children to get a say (12-year-old).

Doctors should be able to tell you what is going to happen before it happens, not after (11-year-old).

My wish is that doctors and nurses talk to me (7-year-old).

Cette dernière phrase, d'un enfant de 7 ans, est à l'image de ce que relate Verpaelst [1] dans son manuscrit et qui souligne l'importance de faire participer les enfants dans les décisions les concernant : "Not once did anyone speak to me directly, or ask me anything personal. It was just a mechanical and mechanistic overview of all things dwarfish. (...). And, no one certainly ever thought of the emotional impact this would have on a seven-year-old boy who was already dealing with enough problems of his own. "

D'autres études montrent que plus les enfants font face à des maladies, plus ils sont alertes et peuvent s'exprimer de façon objective sur leurs situations [15]. Ainsi, affirmer que le développement cognitif et moral d'un enfant serait lié à son âge reviendrait à nier les nombreuses recherches [15-17] qui contredisent les théories de Piaget, [16]. Par exemple, les facteurs environnementaux peuvent influencer le développement de chaque enfant ou encore, certains enfants peuvent avoir une compréhension développée de leur processus mental voire autant que les personnes adultes. En 1991, une expérience menée auprès d'enfants de 4 ans a révélé qu'ils étaient capables de rendre compte de leurs états mentaux passés et de leurs pensées du moment [17]. Pour sa part, en 1972, l'anthropologue Bluebond-Langner [15] a démontré que les enfants étaient capables d'une certaine 
acuité d'un niveau « pré-moral ». La plupart des enfants étudiés, des mineurs en fin de vie atteints de leucémie et âgés de 3 à 9 ans, étaient aptes à rendre compte de la sévérité de leur maladie, de l'éventualité d'une mort imminente et ils montraient un intérêt à en discuter. D'ailleurs faire participer des enfants à la discussion leur permettrait de développer des capacités pour la prise de décisions [9].

Une erreur fréquente est de tenir pour acquis que les enfants qui ne s'expriment pas aussi aisément que d'autres, n'ont rien à exprimer ou qu'ils ne sont tout simplement pas des agents moraux. Les adultes pourraient s'interroger sur le mode de communication des enfants et ils pourraient essayer d'interpréter leurs non-dits à l'aide de compétences de différents professionnels, comme les psychologues. Pour illustrer ce propos, prenons l'exemple d'un cas réel [18] relatif à une très jeune patiente de quatre ans et demi dont les parents ont donné leur consentement à la préservation de sa fertilité précédant les traitements de chimiothérapie qu'elle devait recevoir. Au fur et à mesure de la consultation, la jeune fille était souriante puis elle est passée par des mécanismes de défense où elle ne regardait plus personne. Cet exemple montre que même à un très jeune âge, les patients peuvent s'exprimer et qu'il est possible d'interpréter leur mode d'expression [18].

Loin d'être un plaidoyer pour une reconnaissance totale et aveugle de l'enfant comme agent moral, ce commentaire se veut une modeste contribution à l'éveil des consciences au regard de la place de l'enfant dans les décisions médicales le concernant. À trop vouloir protéger l'enfant, nous pourrions l'isoler maladroitement dans un carcan de vulnérabilité excessive. Un carcan qui, dans un cercle quelque peu vicieux, perpétuerait la capacité limitée des enfants à être impliqués dans les décisions.

\section{Références}

1. Verpaelst F. A very embarrassing moment. BioéthiqueOnline, 2015. 4(7).

2. Couture $\mathrm{V}$. La reconnaissance d'autrui comme prérequis à toute forme de consentement et d'assentiment: Une réponse à Verpaelst et Touyz. BioéthiqueOnline, 2015. 4(17).

3. Honneth A. La théorie de la reconnaissance: une esquisse. Revue MAUSS 2004;(23):133-6.

4. Eshleman A. Moral responsibility. In The Stanford Encyclopedia of Philosophy; 2014.

5. Beauchamp TL, Childress, JF. Principles of biomedical ethics. 6th ed., New York: Oxford University Press. xiii, 2009. p.417.

6. Joffe $S$ et al. Involving children with cancer in decision-making about research participation. Journal of Pediatrics. 2006;149(6): 862-868.

7. Hickey K. Minors' rights in medical decision making. JONAS Healthcare Law, Ethics \& Regulation. 2007;9(3):100-4; quiz 105-6.

8. Nacka $F$ et al. Recherche en pédiatrie (1): dispositions juridiques relatives au consentement. Archives de Pédiatrie. 2015;22(9):983-8.

9. Forman EN, Ladd RE. Ethical Dilemmas in Pediatrics: A Case Study Approach. New York: Springer-Verlag. X; 1991. P.142.

10. DeRenzo EG, Moss J. Writing Clinical Research Protocols : Ethical Considerations. Amsterdam; Boston: Elsevier/Academic. xix, 2006. p.300.

11. Melden Al. Do infants have moral rights?, in Whose Child? Children's Rights, Parental Authority, and State Power. Rowman and Littlefield: New Jersey. 1980. p. 199-220.

12. Les adolescents à l'hôpital. Hôpital.fr. Fédération Hospitalière de France; 2015.

13. Affdal $A O$ et al. Le difficile passage à l'âge adulte en matière de soins : l'exemple de l'épilepsie. Archives de Pédiatrie. 2015;22(4):337-42.

14. Pate, MF. Assent and dissent in pediatric progressive and critical care. AACN Advanced Critical Care. 2013;24(4):356-9.

15. Bluebond-Langner M. The Private Worlds of Dying Children. Princeton, N.J.: Princeton University Press. xv, 1978. 282 p.

16. Piaget J. Le jugement moral chez l'enfant. 5e éd. Bibliothèque de philosophie contemporaine. Paris: Presses universitaires de France. viii, 1978. 334 pages. 
17. Gopnik AS, Slaughter V. Young children's understanding of changes in their mental states. Child Development. 1991;62(1):98-110.

18. Bou Nader, K., Approche psychanalytique de la technique de la préservation du cortex ovarien : Le cas d'Éva, quatre ans et demi, in Le corps sexuel de l'enfant, C. psychosomatique, Editor. 2012. 